\title{
Linx
}

Revue des linguistes de l'université Paris X Nanterre

$52 \mid 2005$

Lexique, terminologie, discours

\section{Le monde perdu des dictionnaires de Maurice Lachâtre (3). Le mystère du Dictionnaire des écoles}

\section{François Gaudin}

\section{(2) OpenEdition}

\section{Journals}

Édition électronique

URL : http://journals.openedition.org/linx/172

DOI : $10.4000 /$ linx. 172

ISSN : 2118-9692

\section{Éditeur}

Presses universitaires de Paris Nanterre

\section{Édition imprimée}

Date de publication : 1 juin 2005

Pagination : $31-42$

ISSN : 0246-8743

\section{Référence électronique}

François Gaudin, «Le monde perdu des dictionnaires de Maurice Lachâtre (3). Le mystère du

Dictionnaire des écoles », Linx [En ligne], 52 | 2005, mis en ligne le 27 janvier 2011, consulté le 19 avril 2019. URL : http://journals.openedition.org/linx/172 ; DOI : 10.4000/linx.172 


\title{
Le monde perdu des dictionnaires de Maurice Lachâtre (3). Le mystère du Dictionnaire des écoles
}

\author{
François Gaudin \\ Université de Rouen
}

Les dictionnaires de Maurice Lachâtre (1814-1900) commencent à être un peu moins ignorés depuis quelques années. Ses trois in-quarto, le Dictionnaire universel (18521856), le Nouveau dictionnaire universel (1865-1870) et le Dictionnaire La Châtre (18981907), dont la publication s'est étalée sur un plus d'un demi-siècle, sont sortis de l'ombre et une étude spécifique a été consacrée par Michel Glatigny au Dictionnaire français illustré (1856-1857). Le cinquième reste à découvrir: un Dictionnaire des écoles. Mais ce lexique élémentaire exista-t-il ? La question ne saurait être tranchée.

Nous sommes en 1856. Maurice Lachâtre commence à faire paraître les livraisons du Dictionnaire des écoles, qui ne sera effectivement publié complet que plus tard, semble-t-il. Il est alors en train de publier son Dictionnaire universel, dont les premières livraisons datent de 1852. Cet ouvrage engagé, dont le titre provisoire fut Dictionnaire du peuple, a été examiné le 19 mars 1856 par le Procureur impérial de la Seine qui a conclu qu'il paraissait «difficile d'intenter des poursuites». Dans une logique éditoriale fréquente en lexicographie, Lachâtre a commencé, en avril 1855, à commercialiser les livraisons, d'un autre recueil lexicographique, le Dictionnaire français illustré, première adaptation de l'ouvrage princeps, en deux volumes d'un format grand in-octavo. Le Dictionnaire des écoles est donc le troisième de la série. C'est le seul monovolume parmi les dictionnaires de Lachâtre. 


\section{Description}

Ce petit ouvrage est d'une extrême rareté. Nous n'en connaissons qu'une version, conservée à la Bibliothèque nationale de France, et dans une édition ou réédition tardive : 1868, date figurant en couverture. L'ouvrage est alors commercialisé par les Docks de la librairie, 13, rue Grammont. Rarissime dans les bibliothèques, l'ouvrage est introuvable sur le marché du livre ancien. De plus, l'exemplaire conservé à la Bibliothèque nationale de France annonce deux ouvrages: le dictionnaire proprement dit, vendu 1 franc, et Les Appendices et le Dictionnaire de la réforme, vendu au même prix, les deux étant proposés également reliés ensemble. Le second est complètement inconnu. Toutefois l'engagement en faveur de la réforme de l'orthographe est ici présent, comme pour les deux ouvrages précédents, puisque le dictionnaire est suivi d'un « traité de grammaire française, de réforme de l'orthographe, de grammaire de la réforme orthographique et du dictionnaire de la réforme», par le fidèle collaborateur Casimir Henricy, fondateur d'une Société de linguistique et de la Tribune des linguistes. On retrouve ici une caractéristique du militantisme lexicographique, agissant à la fois pour la réforme de la société et en faveur du changement volontaire de la langue.

Nous parlons d'une édition ou réédition tardive, car le texte de la lettre-préface, adressée à sa fille, est de 1856. Mais aucune édition complète ne semble avoir paru avant la fin des années 1860. La page de titre, qui diffère de la page de couverture, mentionne la date de 1858 et deux lieux de vente : la Société du Panthéon de la librairie, 26, rue de la Reynie, et la Librairie des cinq centimes illustrés, logée à l'adresse de l'imprimerie Serrière et compagnie ${ }^{1}$. Ces entreprises durent être des relais utilisés par Lachâtre au début de son exil et pourraient être des lieux de vente des premières livraisons, dont la publication aurait été suspendue en raison des démêlés judiciaires de l'auteur-éditeur. La date mentionnée en couverture (1868) correspondrait à la version achevée qui n'aurait donc pu être éditée à la période prévue initialement et n'aurait vu le jour qu'après le premier exil de l'auteur qui dura de 1858 à 1864 .

C'est ce que semble confirmer la lecture de l'article Ortografie, rédigé dans les années 1890, du Dictionnaire-Journal, autre ouvrage, assez curieux du reste, de Maurice Lachâtre, dans lequel on peut lire, sous la plume de l'énigmatique «M.O.» :

Maurice La Châtre s'est déclaré partisan de la réforme orthographique complète, depuis de nombreuses années. En 1868, il publiait un Dictionnaire des Ecoles, à l'usage des enfants et des adultes illettrés, où chaque mot se trouvait écrit selon les règles, et, en regard, orthographié rationnellement ${ }^{2}$.

\footnotetext{
${ }^{1}$ L'imprimeur Serrière faisait partie des opposants au coup d'État de Louis-Napoléon Bonaparte, en décembre 1851, cf. Histoire d'un crime de Victor Hugo.

2 Maurice La Châtre, Dictionnaire-Journal, Paris, Librairie du progrès, tome II, pp. 69-70.
} 
Le mystère du Dictionnaire des écoles

\section{Une dédicace discutée}

Nous avons mentionné la dédicace à sa fille Amélie. Ce texte mérite quelques commentaires, car, d'une part, il éclaire les conceptions de l'auteur - dont on possède peu d'écrits personnels - et, d'autre part, il fut soumis et commenté par Proudhon dont l'avis nous est parvenu.

Le prétexte de cette épître, que nous reproduisons en annexe, est une simple dédicace; or en fait l'auteur, dans ce qu'il présente comme un "véritable cours d'éducation", expose ses conceptions sur un ton grave en développant des préoccupations qui ne connaissaient guère d'échos ailleurs. Cet écrit est le seul de ce type chez Lachâtre.

En premier lieu, il veut que sa fille privilégie les contacts avec les ouvrières : "Aime les ouvrières, tends-leur la main; console-les dans leurs afflictions, partage leurs joies; qu'une douce intimité te fasse vivre de leur vie ; évite, au contraire, le contact des femmes riches, égoïstes et orgueilleuses.» Et de mémoire familiale, on rapporte que la jeune Amélie de La Châtre était obligée par son père à se rendre l'école en sabots et qu'elle en concevait quelque dépit. Dans cet écrit, ce père autoritaire souhaite également qu'elle remplisse sa mission en travaillant, le travail étant « un devoir », et « une joie, un bonheur, quand il nous offre l'occasion d'exercer nos facultés intellectuelles et nos forces physiques selon nos goûts et nos aptitudes. » C'est pourquoi l'enfant cherchera à s'employer de façon utile et variée.

Elle doit choisir avec le même soin une croyance religieuse ; pour le père, il n'y a qu'une seule religion, «celle qu'on nomme philosophie ou religion naturelle »; elle n'a qu'un précepte : «Fais aux autres ce que tu voudrais qu'ils fissent pour toi, dans la limite des droits réciproques. » Au plan politique, la future citoyenne doit former son jugement par l'étude de l'histoire et adopter le système qui mette en pratique les maximes de Cabet, dont il ne cite pas le nom : «Tous pour chacun, chacun pour tous. - A chacun suivant ses besoins; de chacun selon ses forces.» Les conseils bienveillants dissimulent mal l'identification de l'adulte à l'enfant.

Il donne ensuite à sa fille alors unique - qui n'a que six ans... - son avis sur le mariage, qui « ne doit pas enchaîner deux êtres d'une manière indissoluble »; il lui recommande même de se marier dans « une patrie d'adoption » pour bénéficier de la faculté de séparation et, à tout le moins, d'assurer son «indépendance relative». Malgré son jeune âge, il lui conseille d'envisager la mort avec sérénité car elle "n'est qu'un réveil », « la simple apparence de l'absence ». Il pousse le souci du détail jusqu'à la rédaction du testament et à l'embaumement, «afin de prévenir l'effroyable supplice d'être enterrée vive ». L'identification se révèle encore plus clairement quand le père conseille à sa fille de prendre des dispositions testamentaires qui durent être les siennes, si l'on en croit l'étude de certains articles de dictionnaire à teneur autobiographique :

Dispose des biens que je t'aurai laissés ou de ceux que tu auras acquis, en faveur des établissements philanthropiques, pour la totalité, si tu n'as pas d'enfants, et sauf les legs que ton affection te portera à faire aux personnes qui t'entourent; et, dans le cas où tu aurais une famille, réserve une part d'enfant à partager par tiers entre 
l'administration des hôpitaux de Paris, la colonie de Mettray et la colonie de PetitBourg, où se pratique l'éducation commune, gratuite, obligatoire et professionnelle.

En effet, dans le Dictionnaire universel, la section « Modèle de testament» de l'article testament mentionnait les deux colonies. Et c'est sur la pensée de sa propre mort que l'auteur termine son texte, déclarant à sa fille, qu'au-delà de la mort, il n'en veillera pas moins sur sa fille chérie, sur laquelle son « esprit planera sans cesse.» Et il continue dans le même registre: «Dans le sommeil, ton âme conversera mystérieusement avec la mienne ; j'inspirerai tes pensées, j'écarterai de ton chemin les ronces et les épines, et, au moment où ton pèlerinage devra se terminer, je serai là encore pour recevoir ton âme s'exhalant de tes lèvres. »

Le spiritualisme moralisateur inspire-t-il des réticences à son auteur ? Toujours est-il qu'il ressent le besoin de prendre avis de son ami Pierre-Joseph Proudhon, auquel il envoie une copie de son texte très rapidement puisque la réponse en est datée de ce même mois d'août. A cette dédicace où la religiosité se mêle à un lien fusionnel avec l'enfant, Proudhon va répondre sans excessive aménité. Pour épargner le lecteur, nous ne citons que des extraits :

[...] Vous voulez avoir mon opinion sur l'Epître à Amélie. [...]. Je vais vous la résumer en peu de mots [...] Ce que vous avez essayé n'est rien de moins qu'un résumé de principes de la morale, c'est-à-dire ce qu'il y a au monde de plus difficile, et que l'Evangile lui-même, assisté de tous les philosophes, a à peine ébauché.

$[\ldots]$ je ne puis goûter dans son ensemble votre morale : elle n'est pas la mienne, et ce n'est pas celle que j’enseignerai à mes filles.

Il règne d'abord dans votre épittre un air de religiosité dont il me semble que la raison doit être lasse, et qui est d'autant plus singulier chez vous qu'il se trouve de nombreuses impiétés à l'endroit du christianisme [...].

Un autre défaut de votre morale est de ramper dans le passionalisme et l'affectivité pure, chose que ne corrige point du tout la fameuse maxime: Fais aux autres, etc. Car ce n'est pas tout de faire à autrui comme à nous-mêmes; il faut savoir ce que nous devons faire, et pour cela, savoir qui nous sommes, ce que valent nos sentiments, nos goûts, etc. Il y a fort à dire sur tout cela, et, pour me résumer, je vous avouerai que le développement intégral, bon en lui-même et souhaitable, me paraît en réalité dans la vie fort secondaire. [...]

Je ne vous parlerai pas de vos inclinations communistes: elles sont entrées dans votre esprit par la porte de la sympathie qui est une de vos qualités; elles n'en sortiront que par une plus haute intelligence de la dignité humaine qui vous reste à acquérir. Croyez-moi, cher monsieur La Châtre, il en coûte moins de faire amour ou charité que de faire justice ; et c'est la justice qui est le dernier mot et la plus grande parole de la morale.

Sur le mariage, je vous blâme sincèrement d'en parler à votre fille, surtout si jeune ; je vous blâme de le lui présenter comme le nec plus ultra de la félicité humaine ; je vous 
blâme de lui donner à ce sujet des conseils qui tendent à l'indépendance de la femme et, par là, à l'avilissement du mari. [...]

Enfin, je ne puis que sourire de vos consolations contre la mort, et si vous n'avez pas mieux à offrir à l'esprit de votre fille, le mieux est de laisser ce soin au prêtre. [...]

Pardon maintenant de mes critiques. Je ne vous eusse dit mot s'il n'y allait d'un tiers intéressé. Mais je ne puis laisser passer sans protestations les leçons du père quand il y va du salut de la fille, et je crois en mon âme et conscience que la nourriture spirituelle que vous donnez à cette chère enfant est malsaine.

A vous quand même.

\section{P.-J. PROUDHON.}

Le commentaire de Proudhon est révélateur de leur proximité relationnelle et de leur distance intellectuelle. Il parle de deux hommes qui «s'estiment et s'affectionnent» et avoue son attachement à la fille de l'éditeur: "j'aime votre fille comme les miennes ». Les deux hommes étaient donc proches et se fréquentaient. Mais leurs mentalités sont en fait peu compatibles. Proudhon pourrait accepter le syncrétisme politique de Lachâtre ; sa religiosité anticléricale et moralisante le dépasse. Il est dommage que le père de l'anarchie, qui signe certains articles des deux premiers dictionnaires, n'ait laissé aucun témoignage sur cette collaboration. Car elle aurait éclairé les réflexions qui ont présidé à la conception des ouvrages qu’il voit s'élaborer.

\section{Conception}

Le Dictionnaire des écoles ressemble aux deux recueils précédents par sa conception typographique et éditoriale : il commence par un portrait - un dessin assez mauvais, d'ailleurs - de l'éditeur et il est suivi, outre de la lettre à Amélie, d'une notice expliquant la transcription phonétique utilisée.

L'étendue de la nomenclature est de l'ordre de 37000 mots, la nomenclature incluant des noms propres. Pour établir ce chiffre, nous avons compté les entrées des doubles pages des centaines: 100-101, 200-201, etc. Nous avons répertorié 742 entrées, soit 46, 375 par page. La pagination étant de 814 pages, nous avons compté 810 pages de texte, ce qui nous donne pour estimation le chiffre de 37563, 75 entrées. Cela situe l'ouvrage dans un segment qui correspondrait, de nos jours, à celui du Dictionnaire du français au collège, de Larousse, ou du Micro Robert.

Le programme de ce dictionnaire d'apprentissage comprend une transcription phonétique systématique de tous les mots, selon un système décrit en préface, ce qui donne :

Abat-jour, s.m. (abajūr); Jauge, s.f. (jôje); Jérosolymitain, aine, adj. Et s. (jérozolimitén, ène); Jeudi, s.m. (jōdi), Néanmoins, adv. (néanmüén) ${ }^{3}$. Insistons sur le fait que cette transcription est donnée également pour les noms propres: Abailard. (abélar) ; Jéricho. (Jériqô) ; Johannisberg. (Joanisbèr); John Bull. (Djôné

\footnotetext{
${ }^{3}$ Voir plus loin, des extraits du mode de transcription.
} 
François Gaudin

Bül) ; Néerlande. (Neèrlande). Comparons avec un autre lexicographe qui se fixe un même programme, Napoléon Landais, dans son Dictionnaire classique de la langue française, de 1853 : «rendre la prononciation claire et sensible, au moyen de lettres de pure convention et qui donnent le sonnet et exact du mot $»^{4}$. Ses transcriptions sont empreintes du même systématisme, mais elles ne nécessitent pas une explicitation; aucun tableau ni paratexte ne les accompagne; elles sont données comme suit: JaUGE, s.f. (jôje) ; JEUdI, s.m. (jeudi) ; NÉANMOINs, adv. (né-anmoein).

Cet ouvrage, comme les autres du même auteur, sont des ouvrages à macrostructure simple, les dérivés et composés sont traités de façon autonome et non, comme dans les ouvrages à macrostructure double, en sous-entrées. Les choix en matière de microstructure sont ceux d'un dictionnaire d'apprentissage rudimentaire; les articles sont le plus souvent très courts : page 501, sur les 56 articles, 45 tiennent en une ou deux lignes, indications grammaticales et phonétiques comprises. Du coup, les définitions sont extrêmement succinctes. Certains articles sont un peu plus développés, comme jeu ou utopie, qui occupe dix lignes, alors que communisme et socialisme n'en comptent chacun que quatre. L'attention à la rénovation de l'orthographe se marque par l'inclusion à la nomenclature des articles néographe «qui admet une orthographe nouvelle » et néographie. Les citations sont absentes et les exemples rares. En revanche, on n'a pas totalement supprimé l'attention à la phraséologie, qui motive quelques articles un peu plus développés : sous né, on trouve nouveau-né, mort-né, bien né, premier né. Pour comparaison, le Nouveau dictionnaire illustré de Pierre Larousse ne cite dans l'article équivalent que né pour et bien né ${ }^{5}$ et le Dictionnaire classique, de Landais, ne traite que du mot.

$\mathrm{Au}$ niveau de la macrostructure, si les articles sont de longueur réduite, leur nombre ne s'explique pas par une autocensure: tous les termes cités dans les jugements qui vont condamner le Dictionnaire universel, en 1858, et le Dictionnaire du français illustré, en 1859, sont enregistrés à la nomenclature. En dépit de la destination pédagogique de l'ouvrage, le vocabulaire recueilli n'est expurgé ni des termes relatifs à la vie politique, ni de ceux ayant trait à la sexualité : communisme, socialisme, utopie, sont présents, tout comme dans le Nouveau dictionnaire illustré. On y trouve également clitoris, sensualité, sexe, vagin, vulve; or dans le Nouveau dictionnaire illustré, seul sensualité est inscrit à la nomenclature. Pour sa part, le monovolume de Landais ne censure que clitoris, mais il ignore communisme et socialisme.

Malgré la neutralité d'un traitement nécessairement rudimentaire, la nomenclature est tout de même empreinte de choix idéologiques : on trouve ainsi six termes de la famille morphosémantique de jésuite, alors que Larousse n'en cite que quatre. Citons un article :

Jésuitiser, v. n. (jézuitisé). Faire le jésuite, escobarder, tromper avec hypocrisie.

Dans la lignée du Dictionnaire universel, la réduction en un volume à visée didactique se place dans une optique résolument réformatrice et pédagogique. C’est

${ }^{4}$ Napoléon Landais, «Avertissement », Dictionnaire classique de la langue française, Paris, Didier, libraireéditeur, 1853, 596 p.

${ }^{5}$ Nous avons consulté l'édition de 1886. 
ainsi que l'équipe lexicographique adopte un alphabet phonétique qui est utilisé de façon systématique, même pour les noms propres. La présentation de cette notation est précédée d'un texte intitulé «De la prononciation figurée », dans lequel on précise que l'objectif est de fournir au lecteur « un livre donnant la prononciation exacte de tous les mots ». Et pour justifier ce souci d'harmoniser l'expression sonore des signes, l'auteur stipule qu'il vise à «faire que cette prononciation soit la même dans la France entière » et à la fixer. Il note enfin que cet ajout au programme microstructurel, s'il est utile à tous, "est surtout précieux pour les personnes qui se consacrent à l'enseignement, et pour les étrangers. » La note explicative permet de se faire une idée de la transcription utilisée :

Au sujet des caractères employés pour figurer la prononciation.

EU, fort ou long, est figuré par Ō : epinō, orō, hontō, dō, pō, au lieu de épineux, heureux, honteux, deux, peu; ; et EU, faible ou bref, par Ó : favór, nóf, pór, sól, au lieu de faveur, neuf, peur, seul.

OU est figure par $\overline{\mathrm{U}}$ : amūr, für, jūr, au lieu de amour, four, jour.

Le $C$ dur est partout remplacé par le $Q:$ qave, éqole, quvete, au lieu de cave, école, cuvette; le $C$ doux est également remplacé par le $S$ : ansêtres, séder, sela, sime, au lieu de ancêtres, céder, cela, cime.

$\mathrm{CH}$, articulation forte correspondante de $\mathrm{J}$, est figurée par $\mathrm{J}:$ jar, jeval, jimère, joc, jute, roje, au lieu de char, cheval, chimère, cime, chute, roche.

Le G a toujours le son dur : gérir, gider, dégérpir, gijet, au lieu de guérir, guider, déguerpir, guichet; le G doux est partout remplacé par le J : manjer, jémir, jüre au lieu de manger, gémir, givre.

Le K est remplacé par le Q : quaolin, qilograme, qiosqe, au lieu de kaolin, kilogramme, kiosque.

IL et ILL ( $l$ mouillé) sont remplacés par $L L$, qu'il faut regarder, à l'exemple des Espagnols, comme ne formant qu'une seule lettre : travall, solell, palle, travaller, mervelle, bulle, au lieu de travail, soleil, paille, travailler, merveille, houille.

GN est remplacé par $\mathrm{N}$ : sinal, ano, bane, manifiqe, vinoble, au lieu de signal, agneau, bagne, magnifique, vignoble. [...]

Par son caractère radical et systématique, la notation proposée par les lexicographes n'est pas sans intérêt. Elle se situe dans le droit fil de la réforme proposée en annexe du Dictionnaire universel et se rapproche plus du système proposé par Féline, précurseur qui proposa en 1851 un Dictionnaire de la prononciation, que des notations des autres lexicographes animés d'un souci comparable: Gattel, Napoléon Landais ou Charles Nodier, qui se montrent, au XIX ${ }^{e}$ siècle, les continuateurs de Pierre Richelet. Mais soulignons que cette préoccupation n'est pas partagée par tous : des réserves étaient régulièrement émises sur ce sujet par les lexicographes et, encore 
en 1870, Pierre Larousse « reprendra à son compte les observations négatives de tradition sans tenter d'en tirer profit des quelques résultats positifs déjà acquis $»^{6}$.

Lachâtre et Henricy, qui a sûrement joué un grand rôle dans la confection de l'ouvrage, offrent donc en matière de prononciation, dans les limites restreintes d'un monovolume, un service que peu d'ouvrages comparables de l'époque proposent. Car, parmi les nombreux auteurs d'alors, beaucoup, s'ils ont jugé utile une assistance de type phonétique, « ont hésité et renoncé le plus souvent à passer outre la contradiction élémentaire qui les ferait recourir, pour éclaircissement, à un code ésotérique » ${ }^{7}$. Mais, malgré une généralisation de l'usage d'un alphabet phonétique largement diffusé, cette contradiction, et les préventions qu'elle suscite, ont-elles entièrement disparu de nos jours?

En conclusion de cette rapide présentation, on retiendra que ce petit ouvrage à visée pédagogique et utilitaire se singularise par rapport aux autres recueils de Lachâtre et son équipe. Ce n'est guère un dictionnaire pour les révolutions. Fut-il un dictionnaire pour les écoles et qui les pénétra ? On peut en douter. Il n’est connu que des catalogues de libraires, il fut mis en vente, sans doute; fut-il acheté, utilisé ? Rien ne permet à l'heure actuelle d'en décider.

En évaluer la qualité intrinsèque nécessiterait une étude comparative le confrontant à d'autres ouvrages élémentaires contemporains qui lui soient comparables. Un mystère demeure, celui du sort de cet ouvrage dont on ne trouve guère mention que chez Bernard Quemada et ses successeurs, mais que nul ne semble avoir étudié faute tout simplement d'y avoir accès. Connut-il un échec retentissant ? Pour quelles raisons ? Deux dates, trois adresses et trois établissements figurent, nous l'avons dit, sur l'édition tardive de la Bibliothèque nationale de France ${ }^{8}$ : par quelle(s) maison(s) d'édition fut-il commercialisé ? Autant de questions qui peuvent demeurer longtemps en suspens.

Mais concernant Maurice Lachâtre lexicographe, le mystère et l'ombre ne semblent pas près de s'effacer totalement.

\footnotetext{
${ }^{6}$ Bernard Quemada, Les dictionnaires français..., op. cit., p. 110.

${ }^{7}$ Bernard Quemada, Les dictionnaires français..., op. cit., p. 126.

${ }^{8}$ Voir reproduction en annexe.
} 
Le mystère du Dictionnaire des écoles

\section{ANNEXE \\ À MA FILLE BIEN-AIMÉE \\ AMÉLIE LA CHATRE}

CHÈRE ENFANT,

Je te dédie ce livre, pour qu'un jour tu y retrouves la trace de ma constante sollicitude pour ton bonheur. Puisse cette dédicace, véritable cours d'éducation, te servir de règle de conduite dans l'avenir.

Enfant, tu deviendras jeune fille; jeune fille, tu deviendras femme : à tous les âges de la vie, souviens-toi que nulle parmi les ouvrières, tes sœurs, n'est ton inférieure ; que nulle parmi ces femmes que les préjugés sociaux placent aux plus hauts rangs n'est au-dessus de toi; tu es l'égale de toutes, et toutes sont tes égales. Aime les ouvrières, tends-leur la main; console-les dans leurs afflictions, partage leurs joies ; qu'une douce intimité te fasse vivre de leur vie; évite, au contraire, le contact des femmes riches, égoïstes et orgueilleuses.

Chacun de nous, jeté sur cette terre par un décret de Dieu, a une mission à remplir ; cette mission se traduit par un échange de services réciproques. Personne n’a le droit de réclamer le secours de son frère ou d'user des produits de son travail, s'il n'est disposé à lui prêter aide à son tour et à lui rendre l'équivalent de ses produits, du moins selon la mesure de nos forces. Le travail est donc un devoir, et de plus c'est une joie, un bonheur, quand il nous offre l'occasion d'exercer nos facultés intellectuelles et nos forces physiques selon nos goûts et nos aptitudes. Tu chercheras donc, chère enfant, parmi toutes les professions, tous les états, dans les arts et les métiers, ceux qui auront à tes yeux un degré d'utilité réelle pour tes frères ou tes sœurs et qui te plairont davantage; tu en adopteras plusieurs afin de pouvoir varier presqu'à chaque heure du jour tes occupations, et accroître ainsi les jouissances des autres et les tiennes propres.

Le choix d'une croyance religieuse est d'une haute importance. Notre passage sur cette terre est si rapide, qu'à peine avons-nous eu le temps de nous apercevoir que nous existions que déjà il nous faut songer à nous endormir dans les bras de la mort. Notre mission principale est donc ailleurs qu'ici-bas, ou du moins nous en avons une d'un ordre plus élevé que nous devrons remplir sous une autre forme que celle qui frappe nos sens; mystère profond auquel il n'est pas encore donné à l'homme de répondre, et que néanmoins des milliers de religions prétendent expliquer. Chère enfant, écarte de ton esprit les appréhensions de tourments sataniques réservés à nos âmes, suivant quelques théogonies; pratique le bien en vue du bien lui-même, et non par crainte des châtiments éternels. La meilleure religion est celle qui apprend aux hommes qu'ils sont tous les enfants d'un même Dieu; qu'ils doivent s'aimer comme des frères et pratiquer entre eux l'admirable loi de la fraternité ; la meilleure religion est celle qui nous enseigne à suivre les penchants de notre nature, à donner un libre essor 
à nos facultés, à nos sentiments, à nos goûts, à nos aptitudes, mais en tant que les uns et les autres n'apporteront aucun trouble dans l'existence de nos semblables, ne gêneront en rien l'expansion de leurs propres facultés, et ne feront pas obstacle à la liberté d'autrui. Cette religion, qu'on nomme philosophie ou religion naturelle, n'a qu'un précepte : «Fais aux autres ce que tu voudrais qu'ils fissent pour toi, dans la limite des droits réciproques ». Suis ses enseignements et tâche de les répandre, car c'est encore un devoir de chercher à détruire les erreurs et les superstitions chez nos semblables.

En politique comme en religion, la femme doit se former une opinion; elle a le droit de participer au règlement des destinées du pays. Nos mères les Gauloises avaient place aux conseils dans la cité et dans la famille. Tu chercheras donc à éclairer ton jugement par l'étude de l'histoire, par les rapprochements des divers systèmes politiques, et du donneras la préférence à celui qui te semblera réunir le plus de garanties pour le libre essor des facultés de l'homme et de la femme, pour celui qui rendra le mieux dans la pratique ces belles maximes: "Tous pour chacun, chacun pour tous. - A chacun suivant ses besoins; de chacun selon ses forces ».

Devenue jeune fille, sollicitée par la loi de la nature, tu voudras unir ton sort à celui d'un homme que tu auras jugé digne de ton amour; tu accompliras cet acte solennel qu'on appelle mariage. A ce moment, rappelle-toi les conseils de ton père, si déjà je suis parti pour ces mondes où nous allons revivre. Le mariage ne doit pas enchaîner deux êtres d'une manière indissoluble; si ton pays ne reconnait pas la faculté d'une séparation, cherche une patrie d'adoption où tu puisses faire consacrer ton union sous l'empire de lois plus conformes à la nature humaine. Mieux vaut pour une femme renoncer au mariage que de prendre un maitre dans l'homme qu'elle a choisi, ou d'avoir à redouter de se trouver liée pour toujours à un mari qui, dans un temps plus ou moins éloigné, peut devenir pour elle un objet de profonde antipathie, quelle qu'en soit la cause. Toutefois, si tu crois ne devoir pas tenir compte de ces considérations, songe au moins à assurer ton repos matériel, ton indépendance relative, en choisissant dans les modes d'union la forme du contrat qui présente le plus de sécurité, celle du mariage sous le régime dotal ou mieux encore avec la séparation de biens. Plus tard, si Dieu t'accorde des enfants, élève-les à ton tour en vue de les rendre utiles à leurs semblables, et de contribuer à avancer l'heure du règne de Dieu sur la terre.

Tu es encore sur le seuil de la vie, et déjà je songe à la minute suprême. Chère enfant, que cette idée n'assombrisse jamais ton esprit : la mort n'est qu'un réveil, une absence pour les êtres que nous laissons derrière nous, moins encore, la simple apparence de l'absence, car nos âme, après leur séparation de nos corps, continuent à vivre et à planer autour de ceux que nous aimions et que nous n'avons pas cessé de chérir. Sois donc toujours prête pour ce moment; dès que tu auras atteint l'âge de raison, fais tes dispositions dernières afin de ne pas être prise au dépourvu et de remplir ton devoir tout entier; fais ton testament, déposes-en un exemplaire chez un notaire et gardes-en un double en lieu sûr; écris-le seule, sans l'aide ni le conseil de personne, sous les inspirations de la sagesse et l'esprit dégagé de tous préjugés. Ordonne qu'on procède à l'embaumement de ton corps, afin de prévenir l'effroyable supplice d'être enterrée vive, ce qui, hélas! arrive une fois par semaine dans toute la France, et peut-être plus fréquemment encore! Dispose des biens que je t'aurai laissés ou de ceux que tu auras acquis, en faveur des établissements philanthropiques, pour la 
totalité, si tu n'as pas d'enfants, et sauf les legs que ton affection te portera à faire aux personnes qui t'entourent; et, dans le cas où tu aurais une famille, réserve une part d'enfant à partager par tiers entre l'administration des hôpitaux de Paris, la colonie de Mettray et la colonie de Petit-Bourg, où se pratique l'éducation commune, gratuite, obligatoire et professionnelle. Agissant ainsi, tu accompliras un devoir; tu feras restitution au Peuple d'une partie de ces richesses qui toutes viennent de lui, et qu'une justice rigoureuse te commanderait de lui rendre en entier à l'instant même, si la société venait à recevoir une organisation où tous tes besoins et ceux de ta famille seraient assurés au présent et garantis pour l'avenir. Puis, attends avec calme et sérénité le moment suprême de notre réunion.

Chère enfant, lis quelquefois ces lignes que m'inspirent le soin de ton bonheur, le sentiment de mon devoir, pour diriger ton éducation et te maintenir dans la droite voie. Quand j'aurai cessé d'exister sous la forme actuelle, je n'en veillerai pas moins sur ma fille chérie ; mon esprit planera sans cesse autour de toi. Dans le sommeil, ton âme conversera mystérieusement avec la mienne ; j'inspirerai tes pensées, j'écarterai de ton chemin les ronces et les épines, et, au moment où ton pèlerinage devra se terminer, je serai là encore pour recevoir ton âme s'exhalant de tes lèvres.

MAURICE LA CHATRE

Arbanats (Gironde), le 9 août 1856. 
François Gaudin

\section{RÉFÉRENCES}

Gaudin, F. (2002a), «Lettres à André Clas à propos du Nouveau dictionnaire universel de Maurice Lachâtre ", Cahiers de lexicologie No 80, éd. Champion, p. 43-54.

GAUDIN, F. (2002b), « Maurice Lachâtre en Gironde, du phalanstère aux dictionnaires », dans Le Midi dans la nation française, 126e Congrès national des sociétés historiques, Comité des travaux historiques et scientifiques, pp. 71-77.

Gaudin, F. (2003), « Le monde perdu des dictionnaires de Maurice Lachâtre (1) : le Dictionnaire universel (1852-1856)», Cahiers de lexicologie N 83, 2003-2, éd. Champion, pp. 85-104.

GAudin, F. (2004), Maurice Lachâtre (1814-1900) : Portrait d'un éditeur et lexicographe socialiste, thèse de doctorat en histoire, dir. J.-Y. Mollier, Université Versailles Saint-Quentin-enYvelines, 2 vol. 613 p. +34 p ann.

Gaudin, F. à par., «Le monde perdu des dictionnaires de Maurice Lachâtre (3). Le Dictionnaire La Châtre (1898-1907) », dans Aspects diachroniques du vocabulaire, éd. PUR.

GAUDIN, F. (éd .), à par., Le monde perdu de Maurice Lachâtre (1814-1900), Actes du colloque tenu à l'université de Rouen les 16 et 17 septembre 2003, Paris, éd. Champion.

Lindemann, M. (1988), «Les dictionnaires de Maurice La Châtre », dans La lexicographie française du XVIII e au XX' siècle, colloque international de lexicographie tenu à l'Institut de Langues et de Littératures Romanes, Université de Düsseldorf, du 23 an 26 septembre 1986, Actes publiés par Barbara Von Gemmingen et Manfred Höfler, Paris, Librairie Klincksieck, pp. 141-158.

Meschonnic, H. (1991), Des mots et des mondes. Dictionnaires, encyclopédies, grammaires, nomenclatures, Paris, éd. Hatier, LIV p. +311 p.

PARent, I. (2001), Maurice la Châtre et Henry Oriol: deux éditeurs politiques du XIXe siècle, mémoire de DEA dir. J.-Y. Mollier, 147 p.

Pruvost, J. (2002), Les dictionnaires de langue française, Paris, PUF, «Que sais-je ?» $N^{\circ} 3622$, $127 \mathrm{p}$.

QuEmadA, B. (1968), Les dictionnaires du français moderne (1539-1863). Etude sur leur histoire, leurs types et leurs méthodes, Paris, Didier, «Etudes lexicologiques » 4, 683 p. 